25 'Waveriy Fure, New Yurk

NEW YORK UNIVERSITY

WASHINGTON SQUARE COLLEGE OF ARTS AND SCIENCE

MATHEMATICS RESEARCH GROUP

RESEARCH REPORT No. EM.55

\title{
ASYMPTOTIC EVALUATION OF THE FIELD \\ AT A CAUSTIC
}

\author{
by \\ IRVIN KAY \\ and \\ JOSEPH B. KELLER
}

CONTRACT No. AF-19(122)-42

AUGUST 1953 


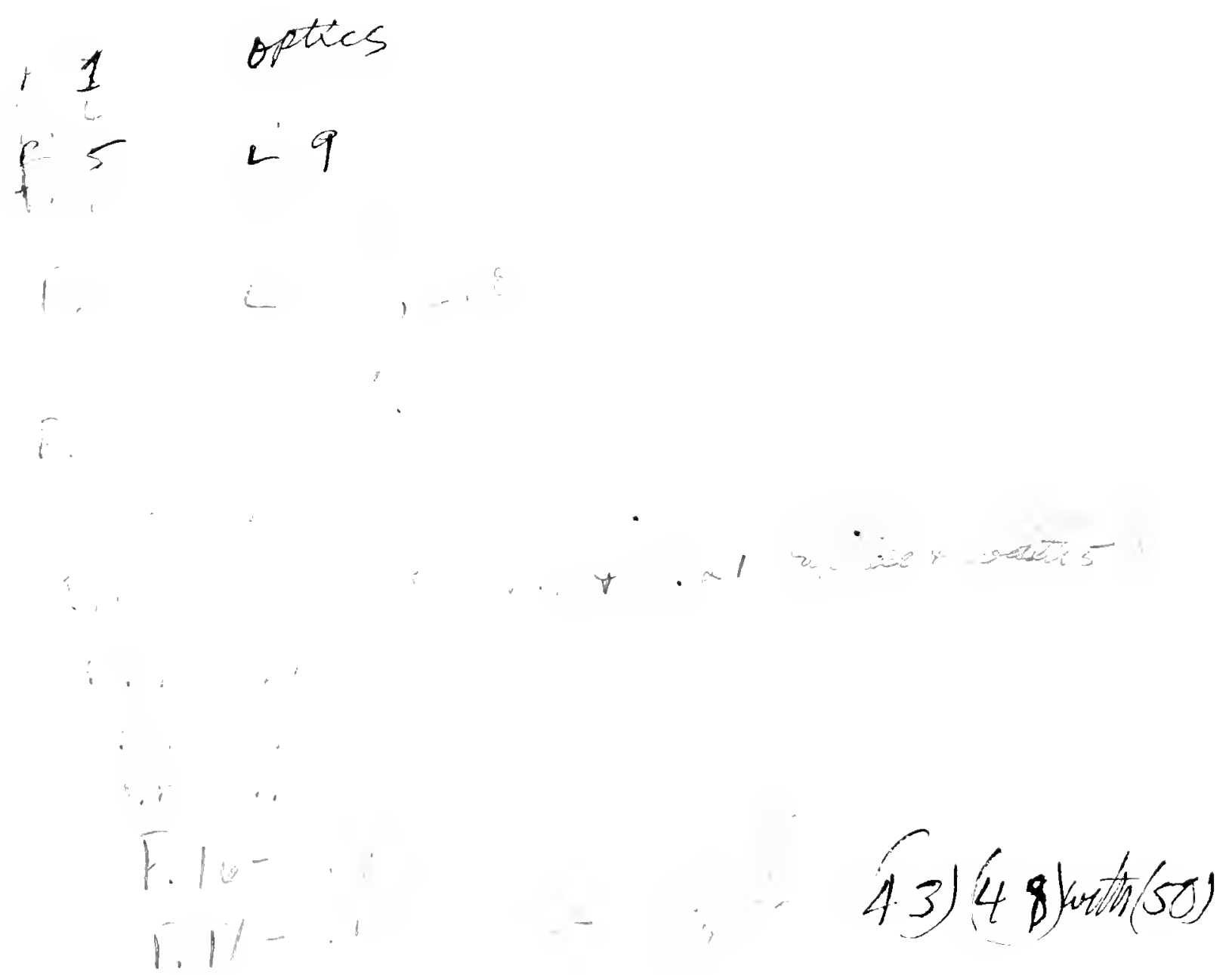


New York Untrersity

Washington Square College of Arts and Sclence

Mathematics Research Group

Research Report No. EM-55

ASYMPTOTIC EVALUATION OF THE FIEID AT A CAUSTIC

by

Irvin Kay

and

Joseph B. Keller
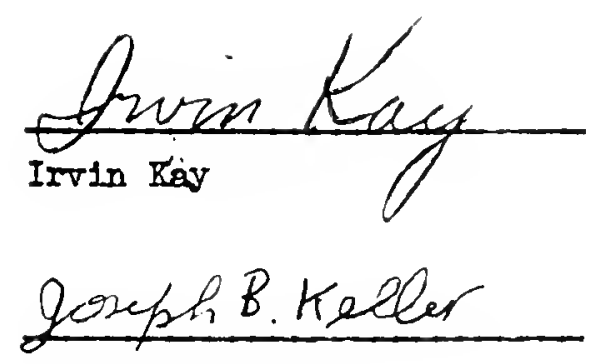

Joseph B. Keller

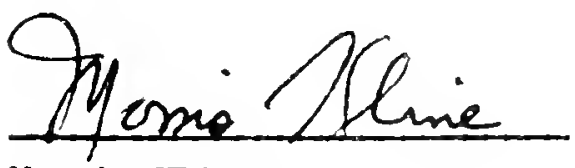

Morris KIine

Project Director

The research reported in this document has been made possible through the support and sponsorship extended by the Geophysics Research Directorate of the Air Force Cambriage Kesearch Certez, under Contrect No. AF-19(122)-42. It is published for technical information only, and does not necessarily represent reconmerdations or conclusiong of the sponsoring agency.

$$
\text { August, } 1953
$$





\section{Introduction}

The electromagnetic field which can be constructed by the methods of geometrical optics, following R.I. Iuneburg [I] becomes infinite at caustics of the optical ray systems. In this paper we overcome this difficulty and obtain a fintte value for the field at a caustic which is produced by the reflection of any incident wave from an arbitrary reflector (all in two dimensions for simplicity). What we actialig deternine is the leading term in the asymptotic expansion of the field with respect to $k=\frac{2 \pi}{\lambda}$, for large $k$. off the caustic this is just the geometrical optics field, but on the caustic it is proportional to $k^{\frac{1}{2}}-\frac{1}{n}$ where $n$ is an integer greater than two. At a perfect focus, such as the focus of a perabolic cylinder, the field is proportional to $k^{\frac{1}{2}}$. Thus on a caustic or at a focus the field increases indefinitely as $k$ does.

We hare exemplified these generai results by considering the special cases of a plane ware incldent on the concare gides of a segment of a parabolic cylinder and a segment of a circular cylinder. In the parabolic case, with incldence along the axis of the parabola, the entire caustic is a point, the focus of the parabola. The asymptotic behavior of the field at the focus is obtained explicitly and transition formulas which show how the field varies in the neighborhood of the focus are also obtained. These formulae inrolve integrals whtch we have evaluated numerically in some spectil cases.

In the case of the circular cylinder, the causic is a curve with a cusp. The asymptotic behovior at the cusp is different from that on the rest of the caustic. The variation of the field near the caustic is described in terms of Harojy's generalized Airy functions.

Since our results yfeld exactly the leading term in the asymptotic expansion of the field, they constitute a check on the solutions proposed by Debye $[2]_{\text {Picht }}[3]$ and Inneburg $[4]$. Each 
of these authors constructed a sclution of the wave equation, or of Manvell's equations, which is regular i: an infinite horogeneous space and satisfies conditions a.t infizity. Iunebure proved that the soluition thus obtained reduces to the known geometrical optics solution as $k$ becomes infinite. In an sctual problem involving boundaries the correct solution aust satisfy certain bouncary conditions. Since the solutions just zentioned do not satisfy such conditions, it is not certain that they will yield the correct field at a caustic in a real problem involving boundaries. However, we have proved that they do yield exectly the save ssymptotic field on a caustic as is givon by our method, and therefore their use in practical problems is justified. 
II. Formulation: The Geometrical Optics Field

We consider the solution $E(x, y)$ of the reduced wave equation

$$
\left(\nabla^{2}+k^{2}\right) \mathbf{E}=0 \text {. }
$$

The solution mist satisfy the boundary conditions

$$
\mathrm{E}=0 \text { on } \mathrm{C} \text {. }
$$

Here $C$ is a given piecewise snooth curve which we will call a reflector." At anp corners of $C$ the derivatives of $\mathrm{E}$ mag become singular. Therefore we must impose a regularity condition at these corners, e.g. that the singularity be the veakest possible one, or that the "energy" integral bo finite. In addition we assume that an incident field $E_{i}(x, y)$ which satisfies ( 1 is given, and that the reflected fleld $E_{I}=E-E_{i}$ satisfies the radiation condition

$$
E_{r}=E-E_{i} \text { is outgoing. }
$$

These conditions complete the formulation of the problem. This formalation applies to the $z$ comonent of the electric field when the reflector is a perfect conductor and the surrounding medium is homogeneous.

Rather than find E itself, we treat the simpler problem of deternining the leading term in the asymptotic expension of $\mathrm{E}$ with respoct to $k$, for large $k$. This is what we call the geonetrical optics field. We begin with the leading term in the asynptotic expansion of the inclient field $\mathbb{B}_{i}$. We assume it to be of the form

$$
\mathrm{E}_{1} \sim \mathrm{A}_{1}(x, y) \text { exp }\left[\log _{1}(x, y)\right]
$$

Now, following Luneburg, we know that the zero-order term in the asymptotic expansion of the field is zero in the shadow. Furthermore, the leading term in the reflected field 18

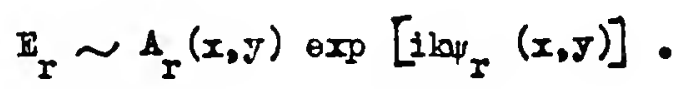

The last statement must be qualified in two respects. First, the form (5) applies only at points not on caustics or shadow boundaries. Second, the leading term will be a sum of expressions of the type given in (5) at points through which more than one reflected ray passes. There will be ono such summand for each ray.

* If $C$ is closed, we consider the solution outside $C$ only, and if $C$ extends to infinity dividing the plane into two parts, we consicier the solution in one part only. 
If we apply (2) to the asymptotic form of $\mathrm{E}$ we obtain

(6) $E \sim A_{i}(x, y) \exp \left[i k_{1}(x, y)\right]+A_{y}(x, y) \exp \left[i k_{y}(x, y)\right]=0$ on $c$.

From (6) we have

$$
A_{x}(x, y)=-A_{i}(x, y) \quad \text { on } C
$$

$$
\psi_{r}(x, y)=\psi_{1}(x, y) \text { on } C \text {. }
$$

(If $\mathrm{E}_{r}$ consists of a sum of terms, the remaining terms occur in pairs satisfying (7), (8), where the indices $i$ and $r$ refer to respective terms of the pair.)

$A_{r}$ and $\Psi_{r}$ off $C$ can be determined by the methods of geometrical optics, using the values on $C$ given by (7), (8). In particular, $\psi_{r}$ satisfies the eiconal equation

(9)

$$
\left|\nabla \Psi_{r}\right|=1
$$

Once $\Psi_{Y}$ is found, $A_{Y}$ can be constructed by the conservation of enerey formula

$$
A_{I}(x, y)=\left(\frac{d \sigma}{d \sigma}\right)^{1 / 2} A_{I}\left(x_{0}, y_{0}\right) \text {. }
$$

Here $x_{0} y$ and $x_{0}, y_{0}$ are two points on $a$ reflectec ray, and $d \sigma_{0} d \sigma$ respectively are cross-sectional leneths of an infinitesimsl tube of rays at each of these points. If we choose for $\pi_{0} y_{0}$ a point on $C$, then (10) determines $A_{r}$ off $C$. If $D$ is the distance along the ray from $z_{0}, J_{0}$ to $x_{,} z$ and if $K_{1}$ is the curvature of the reflected wavefront at $x_{0}, J_{0}$, then it is easily snown that

$$
\frac{a \sigma}{d \sigma}^{1 / 2}=\left(1-D F_{r}\right)^{-1 / 2} \text {. }
$$

On the basis of (5)-(1I) the asymptotic form of the reflected field may be irmediately written as

$$
E_{I}(\pi, y) \sim-\left(I-D K_{0}\right)^{-I / 2} A_{1}\left(x_{0}, y_{0}\right) \exp \left[1 k \psi_{1}\left(\pi_{0}, z_{0}\right)+1 k D\right] .
$$

The point $x_{0} J_{0}$ is the point on $C$ from which the ray through $x, y$ is reflected, and $D$ is the distance between these two points. If there is no reflected ray through $(x, y)$ then $E_{Y}(x, y) \approx 0$. 
When $D K_{0}=1, A_{r}$ becomes infinite. This occurs at the caustics of the reflected reys.

\section{The Field at a Caustic}

In order to obtain a finite value for $\mathrm{E}_{I}$ at a caustic, we begin with Green's formula

$$
E(x, J)=E_{1}(x, y)+\frac{I}{4 i} \int_{C} \frac{\partial E}{\partial n} H_{0}^{(I)}(x D) d s .
$$

Her $\theta, D$ is the distance from the integration point $x_{0}, y_{0}$ on $C$ to $x, y$, and $F_{0}^{(I)}$ is the zero-order Fanicel function of the first kind. To obtain the asymptotic form of $E$ at any point, and in particular at or near a caustic, we insert into the integrand the asymptotic form of $\frac{\partial E}{\partial n}$, which can be computed from (4) and (12). We also replace $\mathrm{H}_{0}^{(1)}$ by its asymptotic form and then evaluate the integral asymptotically. In tiris way we get back the expression for $\mathrm{E}_{\mathrm{r}}$ given by (12) at a.l points off the caustic, as we must expect. At the caust1c, however, we obtain a finite result ratier than the infinite vaiue given by (12). To Justify the method describod we must assume that the caustic does not intersect $C$. Furthernore the lower-order tering in the expansion of $\frac{\partial \mathrm{I}}{\partial n}$. which have been neglected, must not contribute terms to the integral which are of the same (or higher) order as the contribution of the leading terns. That they do not can easily be proved to be the case if reasonable hrpotheses are made about the onitted terns, but this will not be considered here.

We 111 now carry out the calculations just described. When $\frac{\partial E}{\partial n}$ is computed from (4) and (12) and the assmptotic form of $\mathrm{H}_{0}^{(1)}$ is employed, equation (13) yields

(14) $I_{I}(x, y) \sim x^{1 / 2}(8 \pi)^{-1 / 2} \int_{C} A_{1}\left[\frac{\partial \psi_{1}}{\partial n}-\frac{\partial \psi_{T}}{\partial n}\right]^{-1 / 2} \exp \left[i k\left(\psi_{1}+D\right)-\frac{i \pi}{4}\right] d \theta$. 
This integral can be evaluated asymptotically by the method of stationary phase. The phase $\psi_{1}+D$ is a function of the integration point on $C$, and can be considered as a function of arc length $B$ alone $C$. The points of stationary phase are determined by

$$
\frac{d \psi}{d s}+\frac{d D}{d s}=0
$$

Then if $\frac{d^{2}}{d s^{2}}\left(\psi_{i}+D\right) \neq 0$ at the stationary point, (i4) yields

$$
\begin{aligned}
E_{I}(x, y) \sim & \frac{A_{1}}{2}\left(\frac{\partial \psi_{1}}{\partial n}-\frac{\partial \psi_{I}}{\partial n}\right) D^{-1 / 2}\left|\frac{d^{2}}{d B^{2}}\left(\psi_{1}+D\right)\right|-1 / 2 \\
& \exp \left[i k\left(\psi_{1}+D\right)-\frac{1 \pi}{4}\left\{1-\operatorname{sgn} \frac{d^{2}}{d s^{2}}\left(\psi_{i}+D\right)\right\}\right] .
\end{aligned}
$$

In (16) ali quantities on the right side are to be evaluated at the point of stationary phase determined by (15). If there is no stationary point the right side is to be replaced by zero; if there is pore thon one stationary point, the right side is to be a sum with ono term of the above type for each such point.

It follows from (15) that at the stationary points either the law of reflection is satisfied or the reflected ray is the continuation of the incident ray. In the latter case, evaluation of (16) shows that $E_{I} \sim-E_{i}$ so that $E \sim 0$. This occurs in the shadow. In the former case (16) is found to colncide with (12), as is to be expected. It is to be noted that the phase jumps by $\frac{\pi}{2}$ across the caustic.

$$
\text { The expression (16) is not valid when } \frac{d^{2}}{d s^{2}}\left(\psi_{1}+D\right)=0 \text {. }
$$

But if for a polit $x_{0} y$ this second deritative is zero ond also the first derivative (15) is zero, then it can be shown as follows that tinc point Ifes on a caustic oi the reflected raj. $\psi_{1}+\mathrm{D}$ is a fritction of $x, y, s$, say $\varepsilon(x, y, 8)$. 
For each value of s the equation $g_{\mathrm{g}}(I, \mathrm{~g}, \mathrm{~g})=0$, which is (15), defines a ray. If $g_{\mathbf{g g}}(x, y, g)=0$ also, then the point $x, y$ lies on the envelope of the family of rays, which is the caustic.

In order to determine the location of the caustic, we compute

$\frac{d^{2}}{d s^{2}}\left(w_{1}+D\right)$ and obtain

(17) $\frac{d^{2}}{d s^{2}}\left(w_{1}+D\right)=\left(D^{-1}+K_{1}\right) \cos ^{2} \alpha-2 \mathrm{~K}_{c} \cos \alpha$.

Fere $Z_{f}$ and $K_{c}$ are the curratures of the incident wavefront and of the curve $C$, respectively, at the stationary point. The angle $\alpha$ is that between $\nabla_{\psi_{1}}$ and the normal to $C$ at the stationary point. The expressfon in (17) vanishes when $D$ is given by (see also [5])

$$
D^{*}=\left(2 \mathrm{~K}_{\mathrm{c}}-\mathrm{K}_{1} \cos \alpha\right)^{-1} \cos \alpha
$$

Thus (18) determines the distance $D^{*}$ from $C$ along a ray to the caustic, and thus the curvature $\mathrm{K}_{\mathrm{x}}$ of the reflected wavefront at $C$ is given by $\mathbb{Z}_{\mathrm{r}}=\left(D^{*}\right)^{-1}$. The resuzts $(17)(18)$ are used in showtag that (16) colncides with (12).

To obtain the field on the caustic we must evaluate the integral in (14) differentig. For this purpose we consider the integral W given by

$$
W=\int \Lambda(s) \exp [i k f(s)] d s .
$$

Suppose that at a stationary point s at which $\rho^{\prime}(\overline{8})=0$ we also have 
(20) $f^{\prime}(\overline{8})=p^{\prime \prime}(\overline{8})=\ldots=f^{(n-1)}(\overline{8})=0$.

Then the Taylor series of $f(s)$ becomes

(21) $\quad f(8)=f(\overline{8})+(8-\overline{8})^{n} \frac{\rho^{(n)}(8)}{n !}+\ldots$

Therefore $W$ is asymptotically given by

$$
W \sim A(\bar{s}) \exp [\operatorname{likf}(\overline{8})] \int \exp \left[1 k \frac{f^{(n)}(\overline{8})}{n !}(s-\bar{s})^{n}\right] \text { ds. }
$$

Thus we finally obtain

$$
W \sim \Delta(\bar{s}) \exp [i \operatorname{lef}(\bar{s})] 2 k^{-\frac{1}{n}} \Gamma\left(1+n^{-1}\right)(n !)^{\frac{1}{n}}\left|\rho^{(n)}(\bar{s})\right|^{-\frac{1}{n}} \Sigma_{n} .
$$

where

$$
F_{n}=\exp \left[\left(i \frac{\pi}{2} n\right) \operatorname{sgn} \hat{i}(0)(\bar{B})\right] \quad \text { if } n \text { is even }
$$

$$
=\cos \frac{\pi}{2} n \quad \text { if } n \text { ts oda. }
$$

Let us apply the reeult (23) to the integral (14) when the point $x, y$ lies on a caustic and when the first $n-1$ derivatives of $\psi_{1}+D$ vanish at the stationary point. Then we have

(25) $E_{Y}(x, y) \sim v^{\frac{1}{2}}-\frac{1}{n}(n !)^{\frac{1}{n}} \Gamma\left(1+n^{-1}\right)(\pi D)^{-\frac{1}{2}} \sqrt{2}\left|\frac{d^{n}}{d s^{n}}\left(\psi_{1}+D\right)\right|^{-\frac{1}{a}} \cos \alpha$

$$
\exp \left[i k\left(\psi_{1}+D\right)-1 \frac{\pi}{4}\right] \mathbb{F}_{n}
$$

where 
(26)

$$
I_{n}=\exp \left[\left(1 \frac{\pi}{2^{n}}\right) \operatorname{sgn} \frac{d^{n}}{d s^{n}}\left(\psi_{1}+D\right)\right] \quad \text { if } n \text { is eren }
$$

$$
F_{n}=\cos \pi / 2 n \quad \text { if } n \text { is odd. }
$$

It is of interest to notice that the power of $k$ which appears, nemely $k^{\frac{1}{2}-\frac{1}{n}}$, is always a positive power on a caustic since $n \geq 3$. Thus the field increases indefinitely as $\mathrm{k}$ does, The larger the value of $\mathrm{n}$ the higher is the power of $\mathrm{k}$, as one should expect because as $\mathrm{n}$ increases the phase of the integrand becomes more constant. If the phase is exactiy constant, then the power $k^{1 / 2}$ occuris, as will be seen in the example of the parabolic cylincier.

IV. Comparison Hith the Debve - Picht - Iuneburs Solution

In their treatments of the rield at a focus or caustic. Debye, Picht and Irneburg each construct an exact solution of the wave equation, or of Maxwell's equations, which 1s regul of in the infinite homogeneous space. Debye, Picht and Iuneburg determine their solution by recuiring it to have a specified bchavior at infinity, and Inneburg prores that 1 t must reduce to a specified eemetrical optics field as $\mathrm{k}$ becores infinite. In most physical problens the field must satisfy boundary conditions on certain surfaces, and satisfy the wave equation or Mexwell's equations in a partly inhomogeneous mediun. Those conditions are not satisfied by the Dobye - Picht - Luneburg solution. Howerer, this selution does have the same beharior at infinity and the serne ceometrical optics field as the corresponding thysical protlen.

The qucstion therefore arises as to whether the Debye - Picht Innebure solution implies that the field has the same asymptotic behavior at a caustic as that of the correspondine solution of the physical problein.

By the consideretions of Section III (first and second paragragha) both of these solutions, and in fact any solution with the correct geometrical optics field, will imply the correct behavior at caustics and foci pro- 
vided that the lower-order terms in the integrand of (13) do not contribute higher-order terms. Thus all Debye - Picht - Innoburg solutions, although represented by less conventent integrals, will lead to the same leading asyatpotic term at a caustic as does equation (14).

V. Applications: 1. The Parebolic Crlinder

Consider a cylinder, the cross section of which is a segment of a perabola (see Figure 1). Let its focus be the origin of a Cartesian coordinate syster, Its axis be the $x$-azis, and its equation be

$$
z^{2}=-4 p(x-p) \quad x \geq a
$$

As is indicated in the above equation, the segment of the parabola under consideration lies to the right of $x=\alpha$ and 1 s concare toward the left, since we assume that the focal length $p$ is positive. We now suppose that a plane wave is incident from the left with its direction of propagation parallel to the $x$-axts. We as sume that this wave is given by

$$
E_{1}=e^{1 k x}
$$

The incident rays, which are the orthogonal trajectories of the incident wavefronts $x=$ constant, are obviously the lines $y=$ constant parallel to the $x$ - axis. Of these rays all those which intersect the parabolic segment will be reflected through the focus, and therefore the reflected wavefronts are segments of circles with the focus as orlgin. On the basis of this fact and equation (12), we have for the singly reflected field $E_{I}^{(I)}$ 
- 10a -

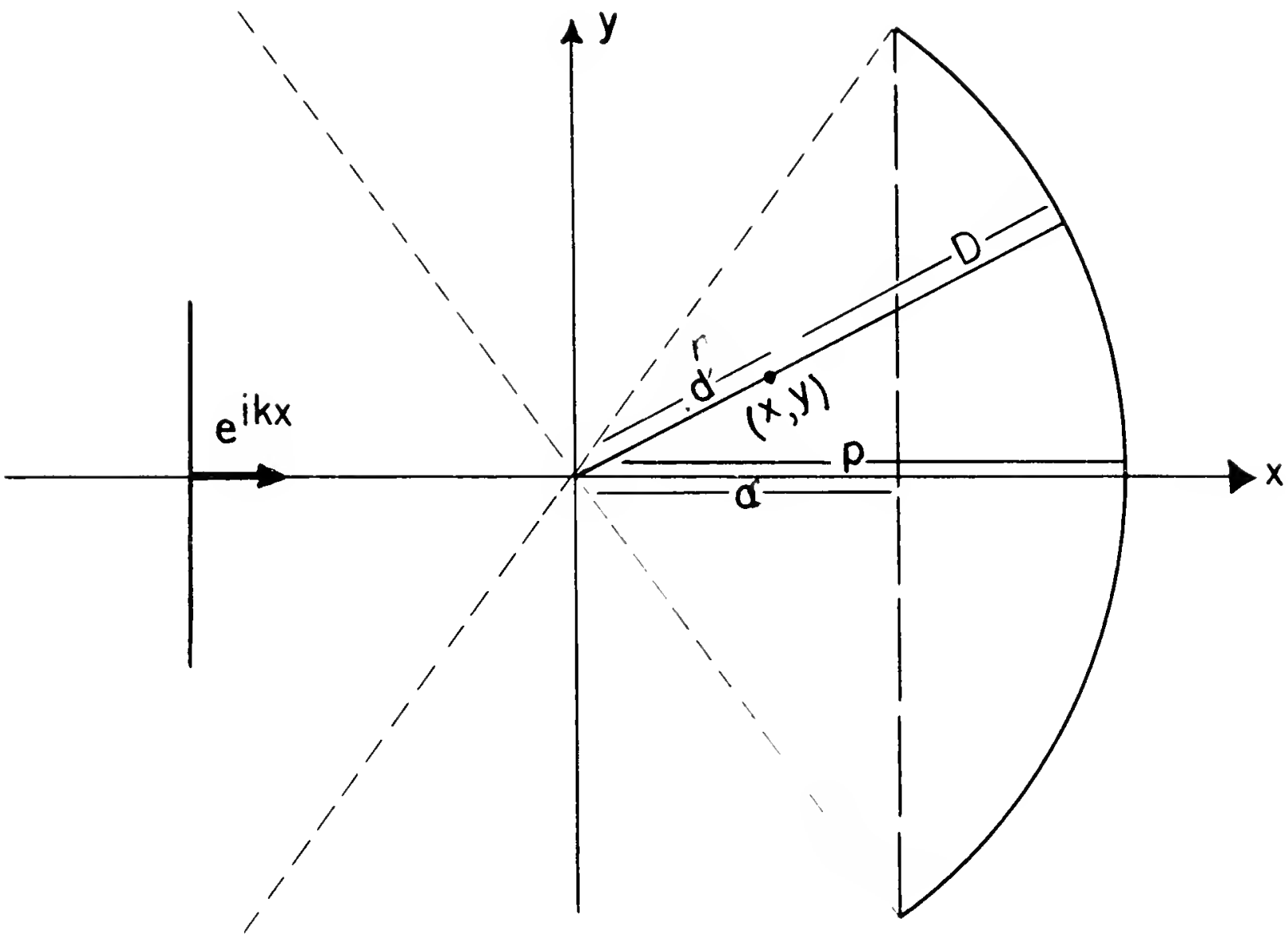

Figure 1 
(29)

$$
\begin{aligned}
& \mathbb{E}_{I}^{(I)}(x, y) \sim-\sqrt{\frac{D+I}{I}} e^{1 k(2 p-r)} \text { before focus, } \\
& \mathbb{I}_{Y}^{(I)}(x, y) \sim-\sqrt{\frac{D-Y}{I}} e^{1 k(2 p+r)-1 \pi / 2} \text { after focus. }
\end{aligned}
$$

In equation (29) I is the distance of the point $x, y$ from the focus and $D$ is its distance along the reflected ray from the point of reflection on the parabola. The phase is easily determined since all rays reach the focus with the same phase 1k2p.

Some rays intersect the parabolic segment a second time after having been reflected through the focus. In order to distinguisin these rays, we introduce the angle $\theta$ between the oncereflected ray and the $x$ - axis. We also introduce the angle $\gamma$ between the $x-$ axis and the line from the focus to the upper end of the parabolic segment. If $\gamma<\frac{\pi}{2}$ then $\alpha>0$ and the entire segment lies to the right of the focus. In this case no ras is twice reflected. On the other hand, if we consider the complete parabola, then $\gamma=\pi$ and every ray is twice reflected, except the ray inc1dent along the $x-a \times 1 \mathrm{~s}$ and characterized by $\theta=0$. In all other cases $\left(\frac{\pi}{2}<\gamma<\pi\right)$ all reys are twice reflected except those satiofying

$$
\pi-\gamma>0>-(\pi-\gamma) \text {, }
$$

which are once reflected.

After the second reflection, the reflected ray becomes parallel to the $x-a x i s$, and the doubly reflected field $\mathrm{E}_{x}^{(2)}(x, y)$ is found from the equations (12) and (29) to be given by

$$
\mathbb{E}_{\mathrm{r}}^{(2)}(x, y) \sim \frac{2 p}{\mathrm{y}} \quad \mathrm{e}^{1 \mathrm{k}(4 p-x)-1 \pi / 2} .
$$


The complete geometrical optics ifeld can now be described as zero in the shadow, $\mathbb{I}_{i}$ at points outside the shadow through which no reflected ray passes, $\mathrm{F}_{1}+\mathrm{E}_{I}^{(1)}$ at points through which one rem flected ray passes, $\mathbb{E}_{1}+\mathbf{E}_{\mathbf{r}}^{(1)}+\mathbb{E}_{\Gamma}^{(1)}$ at points through which two singler reflected rays pass, $E_{1}+E_{I}^{(1)}+E_{r}^{(2)}$ at points through which one singly and one doubly reflected ray pass, and $E_{1}+E_{r}^{(1)}+E_{r}^{(1)}+E_{r}^{(2)}$ at points through which two singly and one doubly reflected ray pass.

We now observe that the field $\mathrm{E}_{r}^{(1)}$, as given by (29), becomes Infinite at the focus of the parabola, and that the field $\mathrm{E}_{I}^{(2)}$ as given by (31) becomes infinite alone the axis $y=0$. This latter singularity appears only in the case of the complete parabola, since the doubly reflected ray $y=$ constant suffers it first reflecIon a.y a point on the parabola with the ordinate $\frac{4 P^{2}}{y}$. Thus as $y$ approaches zero, the oralnate of the point of first reflection must become infintte, and this is possible only with the complete parabola. The $x$ - exis is not a caustic in the ordinary sense of an envelope of rays, but the geonetrical optics field becomes infinite there because the enerey between incicient rays with an arbitrarily large separation Is reflected botween rays with an arbitrarily small separation.

We will now assume that the parabolic segrient is finite $(\gamma<\pi)$, and we will obtain a fintte expression for the fleld at and near the focus, which is the only canstic occurrine in this case. To this end we employ (14), hut we need not consider the contributions of $\mathrm{E}_{\mathrm{r}}^{(2)}$ to the integrand since the doubly reflected field contains no ray through the focus and therefore contributes only lower-order terms to the integral.

First we Introduce the parabolic coordinates $\xi, \eta$ defined by $\xi^{2}=\left(x^{2}+y^{2}\right)^{\frac{1}{2}}-x, \quad \eta^{2}=\left(x^{2}+y^{2}\right)^{\frac{1}{2}}+x$ (32)

$$
x=\frac{1}{2}\left(\eta^{2}-\xi^{2}\right), \quad y=\xi \eta \text {. }
$$


In these coordinates the equation of the parabolic segment is

$$
\eta= \pm \sqrt{2 p}, \quad 0 \leq \xi \leq \sqrt{2(p-\alpha)} \text {. }
$$

Now (14) becomes

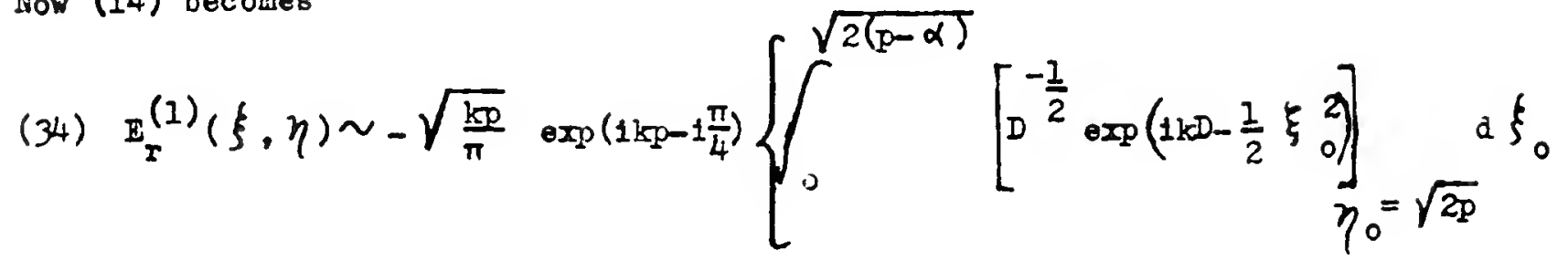

$$
+\int_{0}^{\sqrt{2(p-\alpha)}}\left[D^{-\frac{1}{2}} \exp \left(1 i-\left[-\frac{1}{2} \xi \begin{array}{l}
2 \\
0
\end{array}\right)\right]_{\eta_{0}=-\sqrt{2 p}} d \xi \xi_{0}\right\} .
$$

At the focus this yields

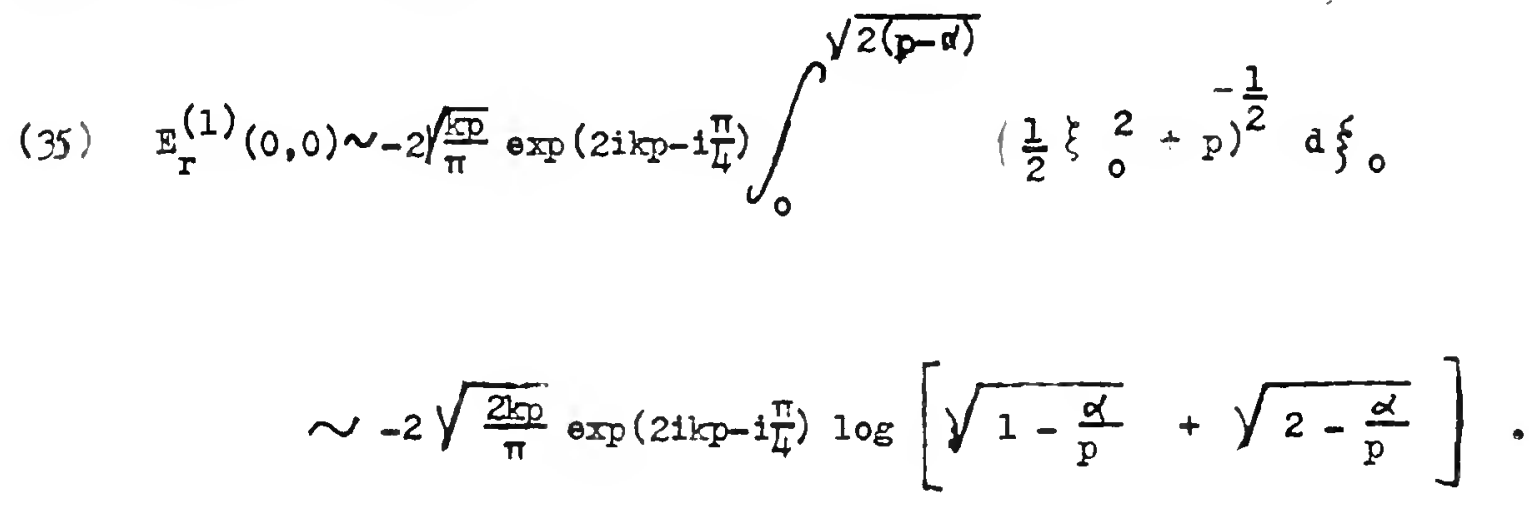

To obtain the field near the focus we introduce the polar coordinates $\rho, \theta$ with the focus as origin and with $\rho$ defined by $\rho=k\left(x^{2}+y^{2}\right)^{\frac{1}{2}}$. Thus $\rho$ is $2 \pi$ times the distance neasured in vaveleneths, of a point froin the focus. Now (34) becomes 
(36)

$$
E_{I}^{(1)}(p, \theta) \sim-\sqrt{\frac{2 k p}{\pi}} \exp \left(2 i k p-\frac{i \pi}{4}\right) \int_{-\gamma}^{\gamma} \sec \frac{\phi}{2} \cdot \theta^{i p \cos (\theta+\phi)} d \phi .
$$

Here $\gamma=\cos ^{-1}\left[\frac{a}{2 p-a}\right]$ is the angular coordinate of the end of the parabolic segment. Figures 2-4, based on eq. (36), show the variation of the reflected field amplitude $\left|\mathrm{E}_{\mathrm{r}}^{(1)}\right|$ along and at right angles to the axis for two different parabolic segments.

2. The Circular Cylinder

A plane wave exp(ikx) is incident on the concave side of the sector of a circular cylindrical zirror of radius $R$. The situation is represented in Figure 5. The center of the mtrror has been placed at the origin of a cartesian coordinate system so that the incident wave wores parallel to the $x$-aris in a positive direction. The engular extent of the mirror is $a+\beta$, where $a$ measures the angle between the $x-e \times 18$ and the end of the sector in the first quadrant, and $\beta$ measures the angles between the $x$-axts and the ond of the segment in the fourth quadrant.

We shail consider only sectors such that no ray is replected more than once. It is apparent from Figure 5 that $\&$ can be at most $\pi-3 a$.

The phase of the incident wave at a point of reflection on the mirror is $R \cos \varphi$, where $\theta$ is the angle of incidence; from Figure it is apparent thet the equation of the reflected ray is

(37) $(R \sin \theta-y) /(B \cos \theta-x)=\tan 2 \theta$; 


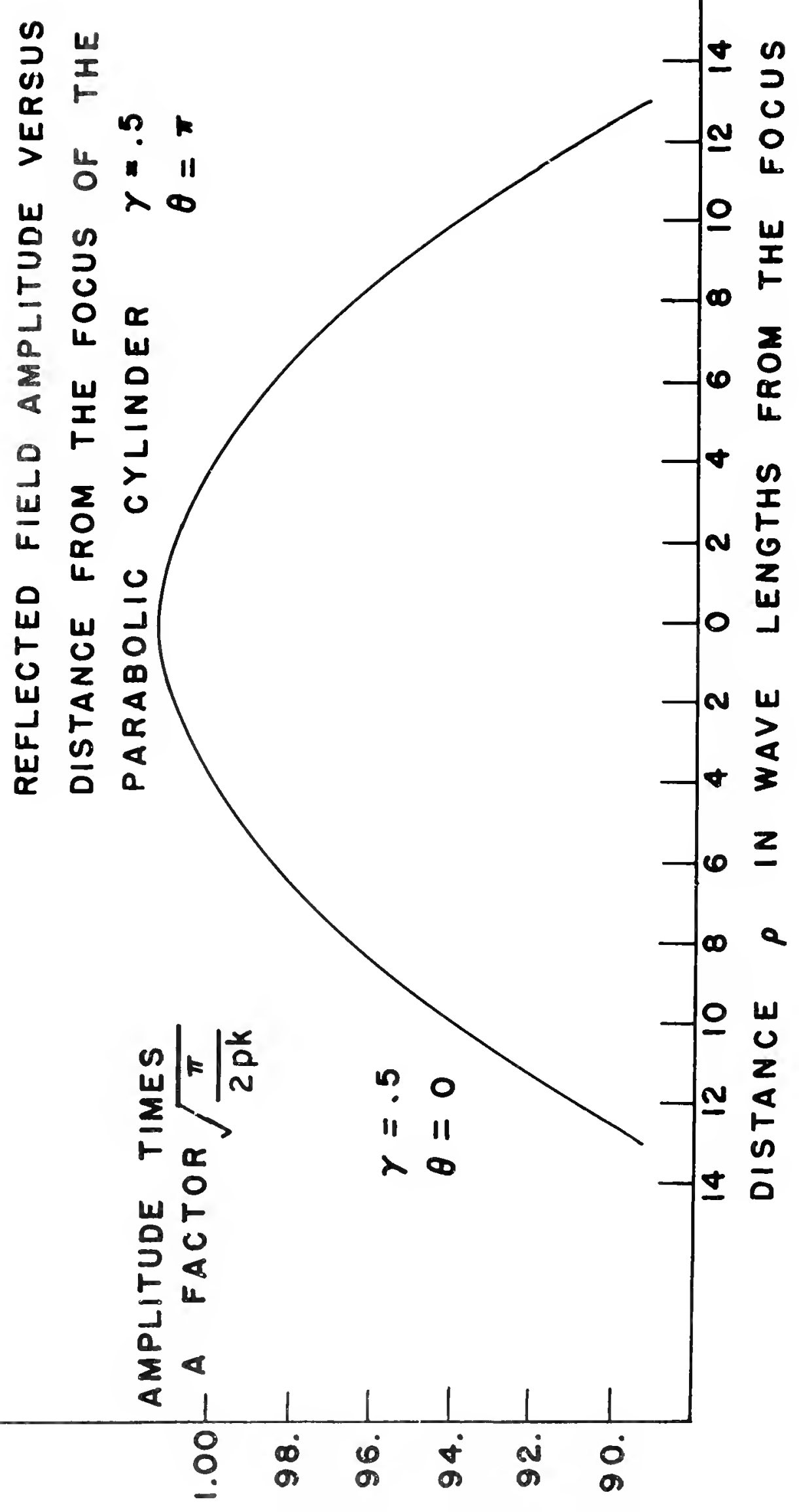




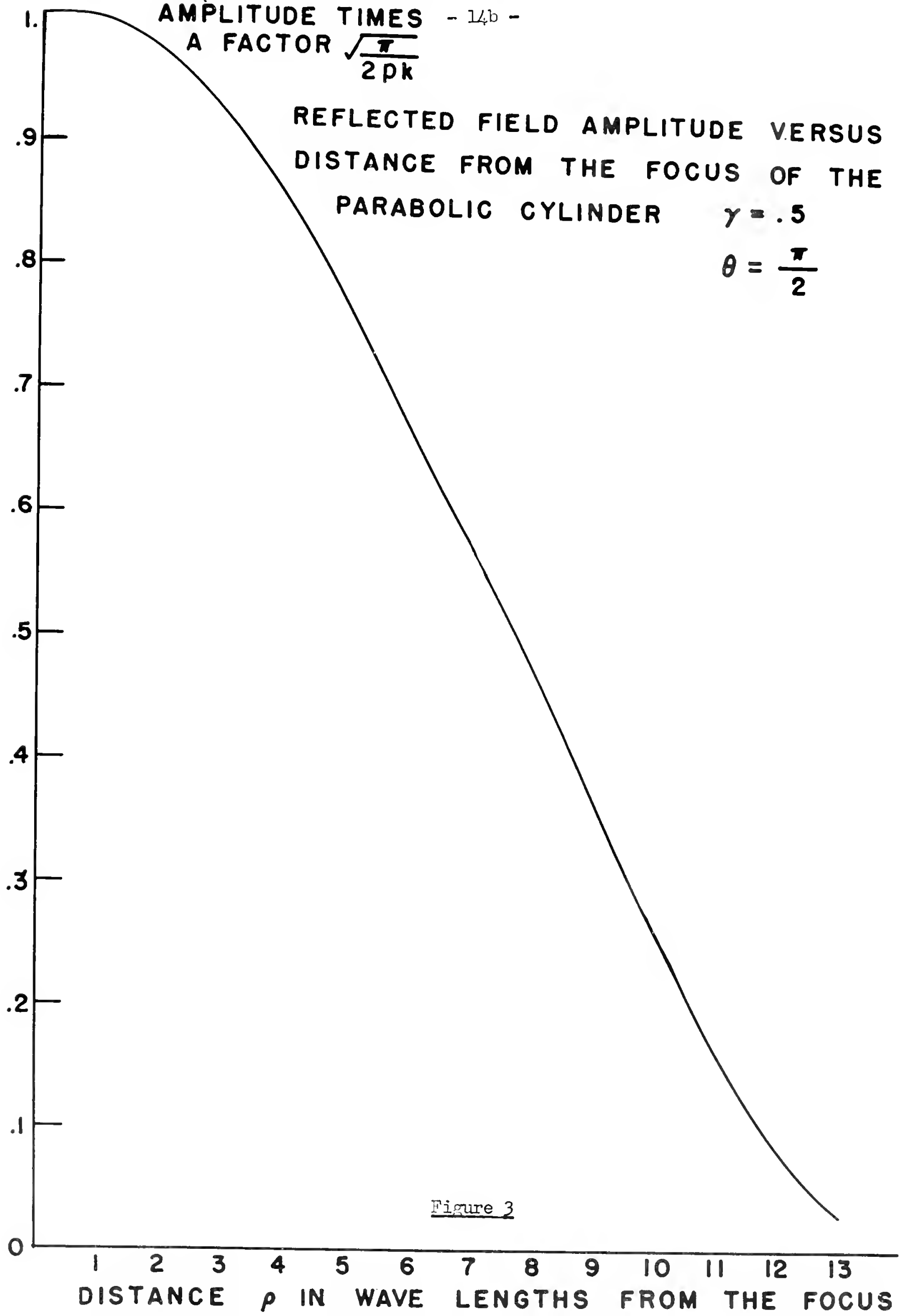




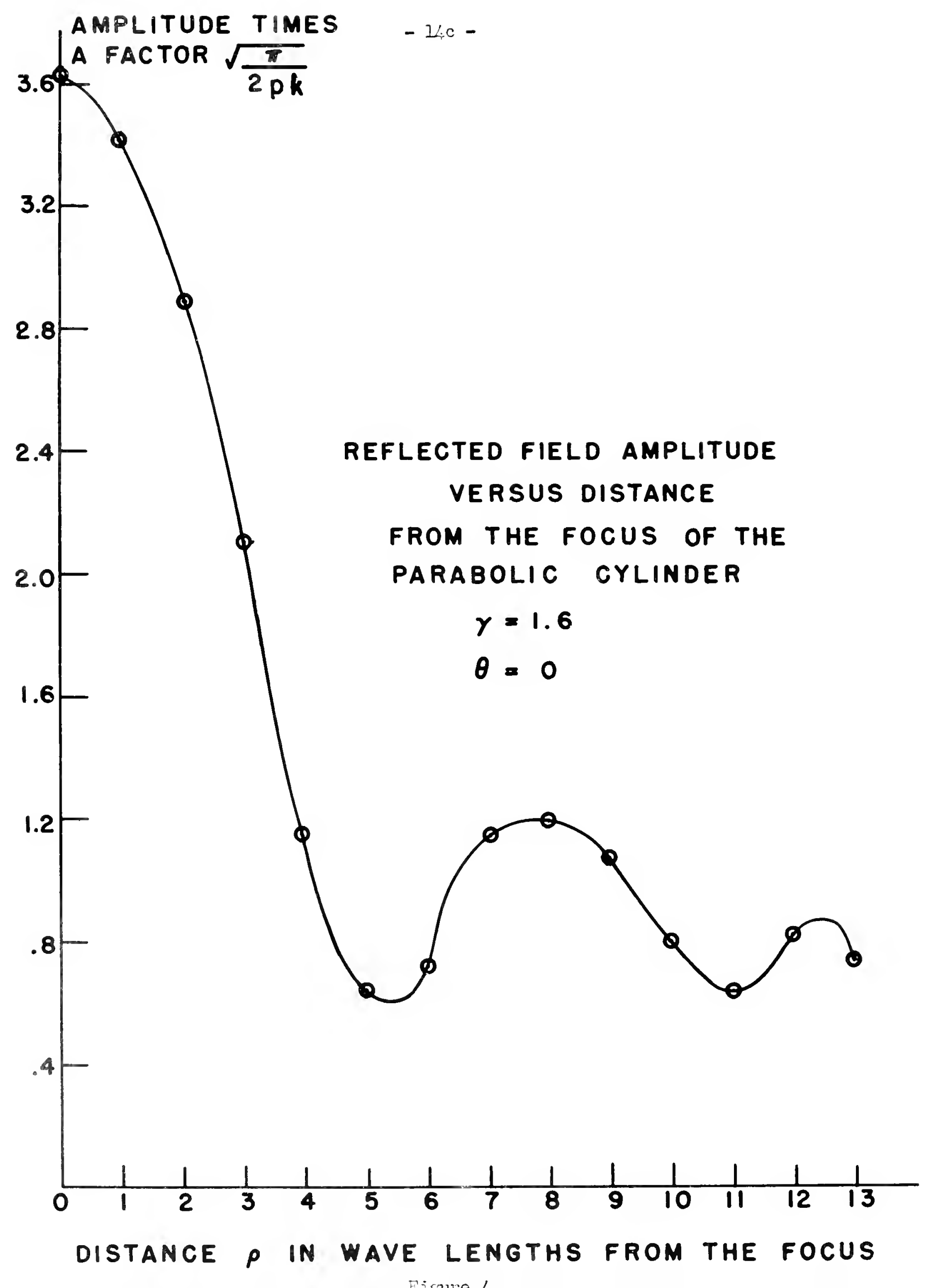


- 1id -

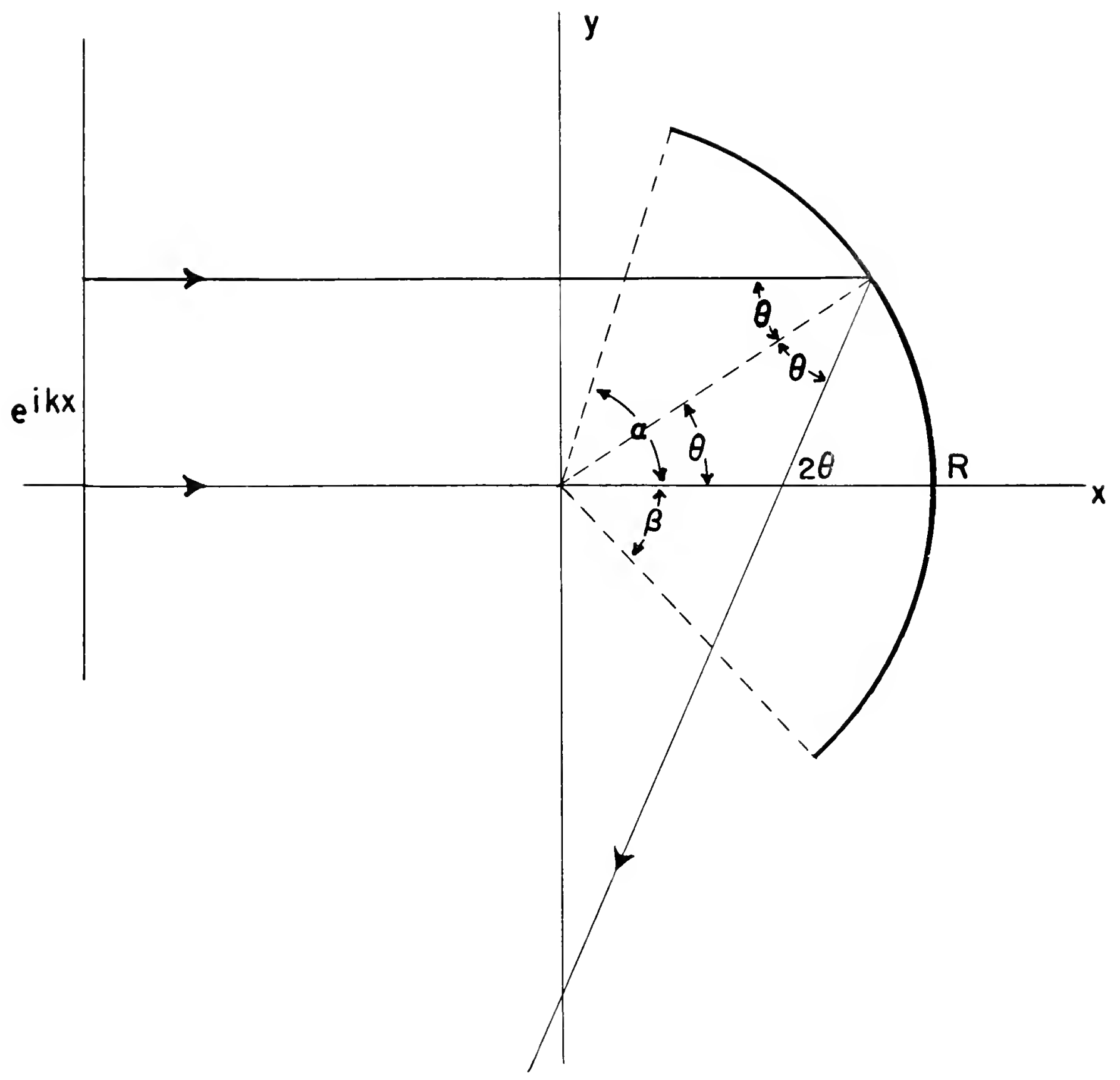

Fimure 5 
$(x, y)$ is an arbitray point on the reflected ray.

Let $D$ be the distance the reflected warefront has traveled from the point of reflection measured along the reflected ray. Then the phase $\psi$ of the reflected wavefront at the point of observation $18 \mathrm{D}+\mathrm{B} \cos \theta$. It 18 clear from Fig. 5 that

(38) $D=(\mathrm{B} \cos \theta-\mathrm{x}) / \cos 20$.

Thus the totel phase at the point of observation 18 given by

(39) $\psi=R \cos \theta+(R \cos \theta-2) / \cos 20$.

By a similar consideration, or from (37), we obtain an expression for $\psi$ involving $y$. Using this expression and (39) we obtain the following parametric equation for a reflected wavefront 1.e., a curve of constant phase $\psi$ :

(40)

$$
x=(R \cos \theta-\psi) \cos 2 \theta+B \cos \theta
$$

$$
y=(B \cos \theta-\psi) \sin 20+R \sin \theta
$$

We can use (40) to obtain a coordinate system for points $(x, y)$ on a reflected ray in terms of the distance $D$ and the angle of incidence $\theta$ by replacing $\psi$ by $\mathrm{R} \cos \theta+\mathrm{D}$ :

$$
x=-D \cos 20+R \cos \theta
$$

(41)

$$
y=-D \sin 2 \theta+R \sin \theta .
$$


Now wo observe that the Jacobian of this transfornation is $2 D-I \cos \theta$, which is zero when $D=\frac{1}{2} R \cos \theta$. This is the distance $D$ to the caustic given by our formula (18):

$$
D^{*}=\left(2 k_{c}-k_{i} \cos \theta\right)^{-1} \cos \theta=(2 / R)^{-1} \cos \theta=\frac{1}{2} R \cos \theta .
$$

We can check this result by calculating the curvature of the reflected wavefront $\psi=$ constant fros $(40)$; we obtain the result $\mathbb{F}_{r}=2(R \cos \theta)^{-1}$ as expected. From our formilas (12) we have for the reflected geometrical optics field the quantity

$$
E_{I} \sim-\left(1-\frac{2 D}{R \cos \theta}\right)^{-1 / 2} \exp [1 k(R \cos \theta+D)]
$$

In terms of the coordinate system defined by (4I).

At the causic, where $D=\frac{l}{2} R \cos \theta$, the reflected field takes a different form, which is found from (25):

(44) $\quad E_{r} \sim(48)^{\frac{1}{3}} \pi^{-\frac{1}{2}} \Gamma\left(\frac{4}{3}\right) \cos \frac{\pi}{6} \cdot k^{\frac{1}{6}} \sqrt{\operatorname{Bcos} \theta}\left|\frac{d^{3}}{d \theta^{3}}(R \cos \theta+D)\right|^{-\frac{1}{3}} \exp \left[\frac{3}{2} i k R \cos \theta-1 \frac{\pi}{4}\right]$.

To calculate the third derivative in this expression we make use of the fact that $\frac{d y}{d \theta}=0$ at the point of reflection and that $D=\frac{I}{2} R \cos \theta$ there. Then

$$
r_{0} \ln \left(0-\theta_{0}\right)=\frac{1}{4} R \sin 20 .
$$

In addition we use the fact that $\frac{\mathrm{d}^{2} \mathrm{y}}{\mathrm{d} \theta^{2}}=0$ when the cioservation point is on the caust1c, ard obtain

$$
r_{0} \cos \left(\theta-\theta_{0}\right)=\frac{R}{2}\left(1+\sin ^{2} \theta\right) .
$$


Then, using (45) and (46), it is easy to find that when the point of observation is on the caustic,

$$
\frac{\mathrm{d}^{3} \psi}{\mathrm{d} \theta^{3}}=-6 \mathrm{R} \sin \theta
$$

Thus the reflected field at the caustic is

(48) $I_{I} \sim 2 \pi^{-\frac{1}{2}} \Gamma\left(\frac{4}{3}\right) \cos \frac{\pi}{6} \cdot(k-R)^{\frac{1}{6}} \cos ^{\frac{1}{2}} \theta \cdot \sin ^{-\frac{1}{3}} \theta \exp \left[\frac{3}{2} 1 \mathrm{kR} \cos \theta-1 \frac{\pi}{4}\right]$.

Mis result holds everywhere on the caustic except at $\theta=0$, where the caustic has a cusp. To obtain the fleld at this point we need one more derivative of $\psi$ at the cusp; this is given by

(49)

$$
\frac{d^{4} \psi}{d \theta^{4}}=-6 R
$$

The reflected field at the cusp is then

(50) $\mathrm{s}_{r} \sim 2^{\frac{3}{2}} \pi^{-\frac{1}{2}} \Gamma\left(\frac{5}{4}\right) \cdot(k R)^{\frac{1}{4}} \exp \left[\frac{3}{2} i k R-\frac{2}{8} i \pi\right]$.

In the neithborhood of the caustic curve there is transition between the various forms of the reflected field given by (44), (48) and (49). To obtain this transition form we must return to the integral expresaion from wich the general reaults wero derived. In fact we write for the reflected field

(5I) $\mathrm{E}_{\mathrm{T}} \sim(2 \pi)^{-\frac{1}{2}} \mathrm{k}^{\frac{1}{2}} \mathrm{D}^{-\frac{1}{2}} \mathrm{R} \cos \theta \exp \left[1 k(R \cos \theta+\mathrm{D})-1 \frac{\pi}{4}\right] \int \exp \Phi \mathrm{d} \theta$. 
(52) $\Phi=\frac{1}{2}+k\left\{\left(D^{-1} R^{2} \cos ^{2} \theta-2 R \cos \theta\right)(\theta-\theta)^{2}+\frac{1}{2} D^{-2} R^{2} \sin 2 \theta(D-R \cos \theta)(\theta-\theta)^{3}\right\}$ weept in the neighborhood of the cusp. In the neighborhood of the cusp we have

$$
\Phi=1 k\left\{\left[\frac{1}{2} D^{-1} R^{2} \cos ^{2} \theta-R \cos \theta\right]\left(\theta^{\prime}-\theta\right)^{2}+\left[\frac{1}{4} D^{-2} R^{2} \sin 2 \theta(D-R \cos \theta)\right]\left(\theta^{\prime}-\theta\right)^{3}\right.
$$

$$
\begin{aligned}
& +\frac{1}{24}\left[R \cos \theta-D^{-1} R(R-D \cos \theta)+4 D^{-1} R^{2} \sin ^{2} \theta-3 D^{-3} R^{3}(R-D \cos \theta)^{2}\right. \\
& \left.\left.+18 D^{-3} R^{3} \sin ^{2} \theta(R-D \cos \theta)-15 D^{-3} R^{4} \sin ^{4} \theta\right]\left(\theta^{\prime}-\theta\right)^{4}\right\} .
\end{aligned}
$$

If we use the value of $\Phi$ given by (53), the integral in (51) can be evaluatec easily in terms of standard functions in the special case where the observation point is the ray reflected through the cusp, the ray being given by $9=0$. On the other hand, if we use the ralue of $\Phi$ given by (52), we can evaluate the integral in (51) with no restriction.

Consider the integral in $(51)$ wit. $\Phi$ given by (52). It has the forrs

(54) $I=\int_{-\infty}^{\infty} \exp \left[i\left(r t^{3}+d^{2} t^{2}\right)\right] d t=|\gamma|^{-\frac{1}{3}} \int_{-\infty}^{\infty} \exp \left[1\left(s^{3}+\delta^{-\frac{2}{3}} \gamma^{2}\right)\right] d \theta$. Let $s=u-\frac{d r^{-2 / 3}}{3}$. Then 


$$
I=2|\gamma|^{-\frac{1}{3}} \exp \left[\frac{2 i \delta^{3}}{27 \gamma^{2}}\right] \int_{0}^{\infty} \cos \left(\frac{u^{3}-\delta^{2} u}{3 \gamma^{4 / 3}}\right) d u,
$$

(55)

$$
=2|\gamma|^{-\frac{1}{3}} \exp \left[\frac{21 \delta^{3}}{27 \gamma^{2}}\right] \mathrm{ci}_{3}\left(\frac{-\delta^{2}}{9 \gamma^{4 / 3}}\right) \text {. }
$$

where $C_{n}(a)$ is Hardy's generalized Airy integral given by Watson [ól :

(56)

$$
C_{i}(a)=\left(\frac{\pi a^{1 / 2}}{6 \sin (\pi / 6)}\right)\left[\frac{J_{-}}{3}\left(2 a^{3 / 2}\right)-J_{\frac{1}{3}}\left(2 a^{3 / 2}\right)\right] .
$$

According to (52) we have

$$
\gamma=\left(k R^{2} \sin 2 \theta / 4 D^{2}\right)(D-B \cos \theta)
$$

(57)

$$
\left.\delta=\frac{1}{2} k\left[R^{2} \cos ^{2} \theta / D\right)-2 R \cos \theta\right] \text {, }
$$

and for the reflected wave field in the neighborhood of the caustic

(58) $E_{x} \sim(2 k / \pi D)^{\frac{I}{2}}|\gamma|^{\frac{-1}{3}} \cos \theta \exp \left[i k(R \cos \theta+D)+1\left(\frac{2 \delta^{3}}{27 \gamma^{2}}-\frac{\pi}{4}\right)\right] c i_{3}\left(\frac{-\delta^{2}}{3 \gamma^{4 / 3}}\right)$.

It is understood of course that (58) holds only when the point of observation is away from the cuap.

$$
\text { If the point of observation is in the neighborbood of the cusp }
$$

and on the line $\theta=0$, we can use (51) and (53) to obtain the reflected wave field in a form similar to (58). Here the integral on the right of (51) has the form 
$-20$

(59) $I=\int_{-\infty}^{\infty} \exp \left[-1\left(\infty 0^{14}+\beta \theta^{\prime 2}\right) \mid d \theta^{\prime}=a^{-\frac{1}{4}} \int_{-\infty}^{\infty} \exp \left[-1\left(t^{4}+\beta a^{-\frac{1}{2}} t^{2}\right)\right] d t\right.$.

We assume $a>0$ in the region considered, since $D \sim \frac{1}{2^{\mathbb{R}}}$ there. Then

(60) $I=a a^{-\frac{1}{4}} \exp \left(i \beta^{2} / 8 \alpha\right)\left\{C_{14}\left(\frac{1}{4} \beta \alpha^{-\frac{1}{2}}\right)-1 s_{14}\left(\frac{1}{4} \beta \alpha^{-\frac{1}{2}}\right)\right\}$.

Thus

(bI) $\left.E_{I} \sim(2 k / \pi D)^{\frac{1}{2}} B_{c i}^{-\frac{1}{4}} \exp \left[i k(B+D)+1\left(\beta^{2} / 8 \alpha-\pi / 4\right)\right] \int_{14}\left(\frac{\frac{1}{4}}{4} \alpha^{-\frac{1}{2}}\right)-1 S_{i 4}\left(\frac{1}{4} \beta \alpha^{-\frac{1}{2}}\right)\right\}$

with

(62) $\alpha=\frac{1}{24}\left[B D^{-1}(R-D)-R+3 R^{3} D^{-3}(B-D)^{2}\right]$

$$
\beta=R-\frac{1}{2} R^{2} D^{-1}
$$

Again tile functions $C_{i 4}$ and $S_{14}$ are lardy's generalized Airy integrals [6]:

$$
c_{1 n}\left(c_{1}\right)=\pi \alpha^{\frac{1}{2}}[2 n \sin (\pi / 2 n)]^{-1}\left\{J_{-1 / n}\left(2 x^{n / 2} ;-J_{1 / n}\left(2 \alpha^{n / 2}\right)\right\}\right.
$$

(63)

$$
S_{\text {in }}(\alpha)=\pi \alpha^{\frac{1}{2}}[2 n \cos (\pi / 2 n)]^{-1}\left\{J_{-I / n}\left(2 \alpha^{n / 2}\right)+J_{I / n}\left(2 \alpha^{n / 2}\right)\right\} .
$$

Equation (61) gives the reflected field in the neighborhood of the cusp provided that the observation point is or the exits of the circular fairer.

Acknowledgments

The authors wish to thank Professor Fritz Reich for his stiuuletin e discussions durphtinis investigation. 


\section{References}

1. Inneburg, R.K., Asymptotic Development of Steady State Electromagnetic Fields

N.I.U., Washington Square College, Mathematics Resecrch Group, Rosearch Report No. FM-14, (1949).

2. Debys, P., Das verhalten von Iichtwellen in der o oine oines Brenopunktes oder ciner Brennlinie Arn. D. Pnys. (4) 20, 755, (1909).

3. Picht, J., Öber den Schwingungsvorgang, der einem beliebigen (astiematischen) Strahlenbiindel entapricht Ann. d. Phys. (4) 27, 685, (1925).

4. Iuneburg, R.K. Asymptotic Evaluation of Diffraction Integrals N.I.U., Washington Square College, Math. Res. Group, Research Report No. EM-15, (1949).

5. Beller, J.B. and Eeller, H.R., Determination of Reflected and Transmitted Fields by Geometrical Optics N.Y.T., EM-13, (1949) or Jour. Optical Soc. Am., Vol. 40, No. I, pp. 48-52, January, (1949).

6. Watson, G.N. A Treatise on the Theory of Bessel Functions The Macnilian Co., New York, (1948), p. $320 \mathrm{ff}$. 



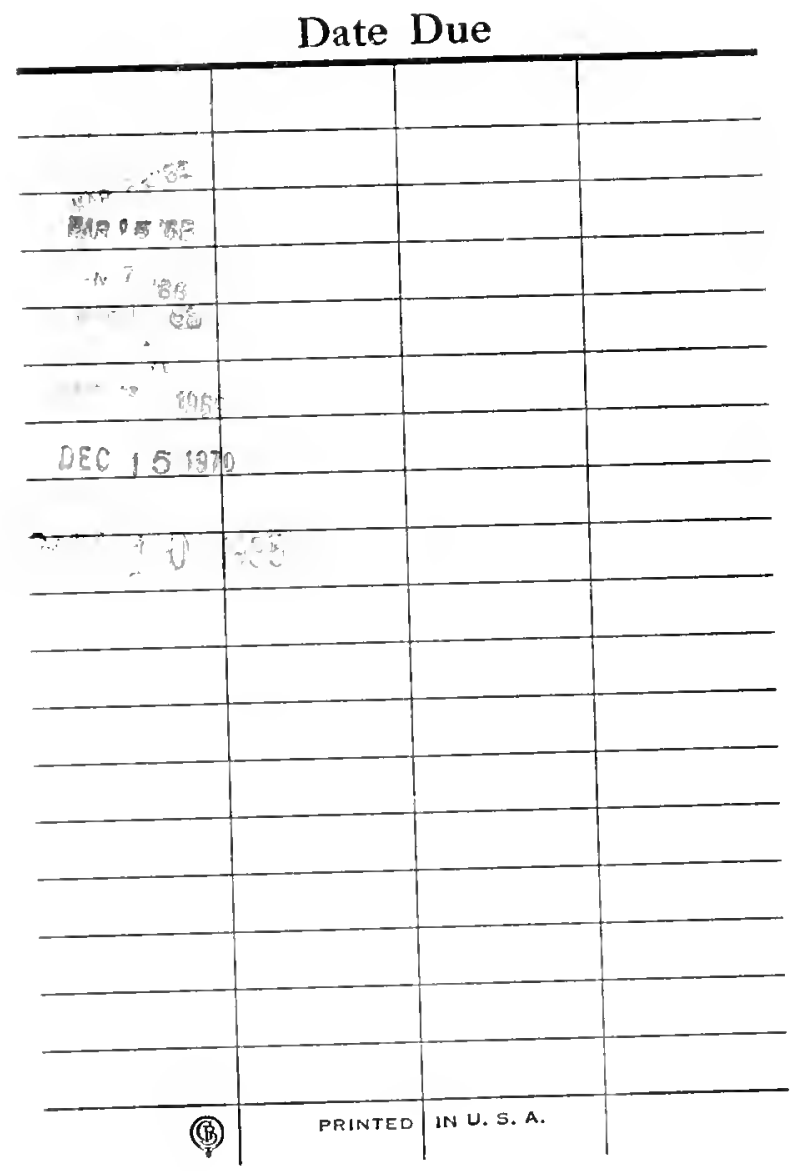




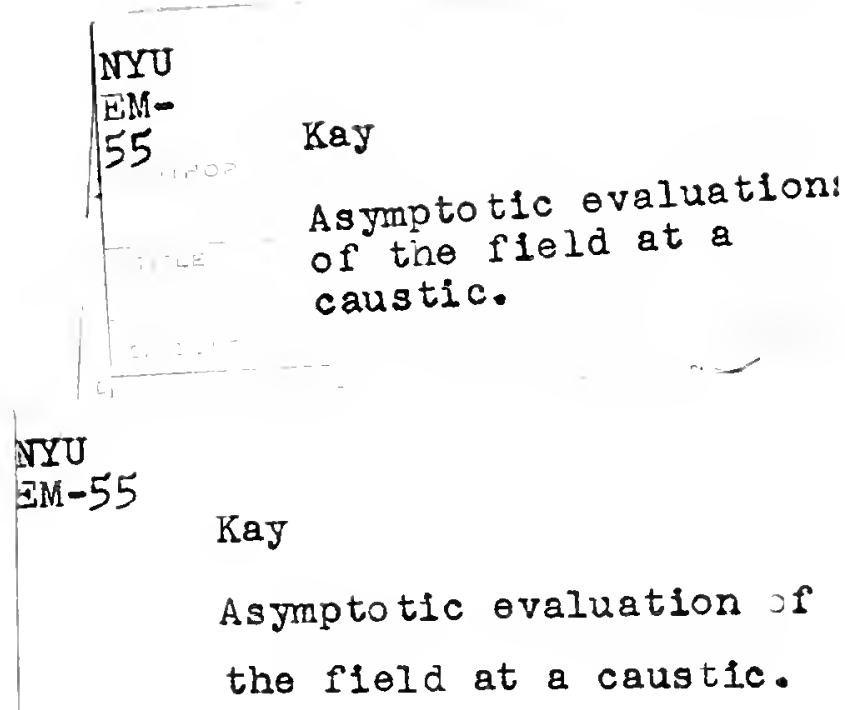


